\title{
Corela
}

Cognition, représentation, langage

$18-2 \mid 2020$

Vol. $18, n^{\circ} 2$

\section{Inégalité(s) dans le discours de la presse française : usages discursifs et dimensions sémantiques d'un mot}

Pascale Brunner et Michele Pordeus-Ribeiro

\section{OpenEdition}

Journals

Édition électronique

URL : https://journals.openedition.org/corela/12798

DOI : $10.4000 /$ corela. 12798

ISSN : 1638-573X

Éditeur

Cercle linguistique du Centre et de l'Ouest - CerLICO

Référence électronique

Pascale Brunner et Michele Pordeus-Ribeiro, «Inégalité(s) dans le discours de la presse française usages discursifs et dimensions sémantiques d'un mot ", Corela [En ligne], 18-2 | 2020, mis en ligne le 30 novembre 2020, consulté le 14 juillet 2021. URL : http://journals.openedition.org/corela/12798 ; DOI : https://doi.org/10.4000/corela.12798

Ce document a été généré automatiquement le 14 juillet 2021.

\section{(c) (i) (3)}

Corela - cognition, représentation, langage est mis à disposition selon les termes de la licence Creative Commons Attribution - Pas d'Utilisation Commerciale - Partage dans les Mêmes Conditions 4.0 International. 


\title{
Inégalité(s) dans le discours de la presse française : usages discursifs et dimensions sémantiques d'un mot
}

\author{
Pascale Brunner et Michele Pordeus-Ribeiro
}

\section{Introduction}

1 Depuis la crise financière de 2008 et la remontée du chômage qui s'est ensuivie, la question des inégalités a été placée sur le devant de la scène (Duvoux $2017: 3$ ), et il n'est pas rare de voir le mot inégalité(s) apparaître dans les discours médiatisés, notamment lorsqu'il s'agit de décrire et d'expliquer des situations de crise sociale ${ }^{1}$. L'inégalité est, par ailleurs, un phénomène qui se mesure et qui se traduit en indicateurs et en chiffres : ainsi, par exemple, selon les données de l'Institut national de la statistique et des études économiques, en France, en 2017, le taux de pauvreté monétaire, au seuil de $60 \%$ du niveau de vie médian, est de $14,1 \%$, soit 8,9 millions de personnes vivant avec moins de 1041 euros par mois (Insee 2020 : 62). L'inégalité se manifeste sous des formes diverses : elle peut ainsi être économique, territoriale, culturelle et sociale; elle peut concerner les différences de salaires, ou bien les différences entre les femmes et les hommes. Mais elle peut aussi apparaître sous-déterminée, la seule présence d'un article défini étant suffisante à l'identification du phénomène, comme dans cet extrait d'un article à caractère économique paru dans Le Monde: "Les États-Unis constituent donc un très bon risque, même si la croissance y est moindre qu'avant crise, la société plus dure et les inégalités plus fortes » (27-01-2015). Enfin, objet de recherche privilégié des sciences humaines et sociales ${ }^{2}$, l'inégalité revient couramment dans les dires des journalistes et dans la communication des femmes et des hommes politiques, de droite comme de gauche, très souvent dans des structures exprimant le souhait de lutter contre, ou le besoin de la réduire, comme dans cet énoncé rapporté : " "Qui s'opposera à ce que le gouvernement grec vienne en aide aux plus pauvres ou veuille 
réduire les inégalités?" explique Pierre Moscovici» (Libération, 19-02-2015), laissant entendre un certain consensus autour de la thématique.

Nous proposons, dans cet article, d'aborder cette question de l'inégalité à travers une réflexion, à la fois sémantique et discursive, sur le mot inégalité(s) - lequel est d'ailleurs chargé d'histoire et toujours d'actualité politique et sociale - à partir d'un corpus de discours de la presse nationale française. L'objectif principal est alors d'établir le sens de ce nom à travers l'examen des constructions et des associations qui apparaissent, de façon récurrente, dans son cotexte. Le corpus sur lequel porte l'analyse est composé d'un ensemble de documents extraits des journaux Le Monde, Le Figaro et Libération, recueillis à partir de la base de données en ligne Europresse; il regroupe tous les textes de ces trois quotidiens parus entre le $1^{\mathrm{er}}$ janvier et le 31 décembre 2015, contenant les mots inégalité et inégalités, et est constitué de 1404 documents, de longueurs variables, appartenant à des rubriques journalistiques différentes (Politique, Société, Environnement, Débat, Livres, etc.) ; le corpus contient 1746 occurrences de la forme inégalité(s) ${ }^{3}$ et sera soumis aux logiciels de statistique textuelle Lexico3 et Le Trameur ${ }^{4}$.

3 L'article s'organise en trois parties. Nous débuterons l'étude par quelques questionnements de nature référentielle et sémantique suscités par des emplois spécifiques du mot dans le corpus de presse, ainsi que par l'examen des définitions de dictionnaire ( $\$ 1)$. Ensuite, nous présenterons brièvement la démarche sur laquelle repose l'analyse du sens d'inégalité(s) en discours (\$2). Enfin, nous exposerons les dimensions sémantiques principales du mot, lequel fonctionne, dans le corpus, comme un fait pluriel, concret et mesurable ( $\$ 3.1$ ), mais aussi comme un fait, qui souvent évalué négativement (\$3.2), peut également être à l'origine de revendications (\$ 3.3). En conclusion, nous reviendrons sur la façon dont ces dimensions se manifestent à travers la matérialité discursive et sur la façon dont elles interagissent entre elles.

\section{Interrogations référentielles et sémantiques}

4 Lorsque l'on choisit de faire porter l'analyse sur un mot tel que inégalité(s), l'attention se tourne d'abord vers des questionnements de nature référentielle. On observe, en effet, que le lien entre le nom et l'entité à laquelle il réfère n'est pas aisé à établir : a-ton affaire à une entité abstraite ou concrète? La présence, dans l'entourage immédiat du nom, d'un modificateur, suffit-elle à identifier son référent? À ces interrogations, on peut ajouter le peu d'éléments fournis par les dictionnaires de langue française, dont les définitions ne reflètent pas l'importance du mot dans les discours sociopolitiques.

\subsection{Un référent qui fluctue}

5 On peut intuitivement penser que le mot inégalité(s), dans la mesure où il renvoie à une entité immatérielle, entre dans la catégorie des noms abstraits. Ce classement, qui met en avant les caractéristiques ontologiques du référent, est conforté par des extraits du corpus dans lesquels inégalité côtoie d'autres noms considérés, eux aussi, comme abstraits, comme en (1), où le mot est dans un rapport d'identité référentielle avec thème, ou en (2), où il est caractérisé comme un sentiment :

(1) Ces initiatives ont suscité un tollé chez les conservateurs, mais il est intéressant de constater que plusieurs candidats présidentiels potentiels, comme Jeb Bush ou 
Mitt Romney, ont mis récemment sur le tapis le thème de l'inégalité. (Le Figaro, 21-01-2015)

(2) Les entreprises doivent fournir un effort constant de neutralité sur ces questions. Autant que le chômage des jeunes, le sentiment d'inégalité et de discrimination est source de colère. (Le Monde, 18-11-2015) réelles et spécifiques, possédant les propriétés de l'inégalité. Ainsi, dans l'extrait (3), inégalité renvoie, non pas à un thème ou à un sentiment, mais à une situation bien délimitée, celle de la différence entre des religions, et, dans le cas de (4), la présence du verbe exister fait ancrer l'interprétation d'inégalités dans le réel :

(3) Les autorités musulmanes se disent confrontées à une situation d'inégalité. La loi de 1905, modifiée en 1907 et en 1908, favorise l'Église catholique. (Libération, 21-06-2015)

(4) Mais l'Afrique n'a pas seulement besoin d'augmenter son niveau de croissance économique. Il faut surtout résoudre les inégalités qui existent entre les pays et à l'intérieur des pays. (Le Monde, 02-09-2015)

7 À travers ces exemples, on voit que le fonctionnement référentiel d'inégalité(s) est fluctuant, le mot pouvant désigner, selon les usages, des entités abstraites (un thème, un sentiment), ou bien des situations réelles et inégales, vécues par un individu ou un groupe d'individus. En tout cas, cette instabilité référentielle ne porte pas préjudice à l'interprétation et ne paraît pas générer de débats autour du sens : comme on l'observe dans cet énoncé à caractère définitoire (5), l'usage de l'expression passe-partout ${ }^{5}$ une chose montre que, pour l'énonciateur, le mot inégalité n'a pas besoin d'être davantage explicité pour être employé et compris par les interlocuteurs et par les lecteurs du quotidien.

(5) L'Écossais [A. Murray], qui a été entraîné à ses débuts par sa mère et a grandi entouré de femmes, a remis ça à Roland-Garros, puis dans la presse : «Je suis pour que tout le monde soit égal et si c'est ça être féministe, alors oui, vous pouvez dire que je le suis. J'ai vraiment ouvert les yeux quand j'ai commencé à travailler avec Amélie [Mauresmo]. L'inégalité est une chose que j'ai commencé à voir et qui me passionne. Ça m'a ouvert l'esprit. » (Libération, 07-07-2015)

8 L'observation du corpus permet de relever d'autres procédures linguistiques fréquentes, comme la détermination du nom par des expressions à fonction classificatrice, point que nous allons maintenant aborder en essayant de répondre à cette question: l'information apportée par l'ajout d'un déterminant suffit-elle à circonscrire le référent d'inégalité(s)?

\subsection{Un champ référentiel divers}

9 Dans les discours, le mot peut, en effet, apparaitre accompagné d'un modificateur, un adjectif ou un syntagme prépositionnel, dont la fonction est de classer le nom selon le domaine dans lequel l'inégalité est attestée (inégalités scolaires, de santé) ou, dans une moindre mesure, selon les entités considérées comme inégales (inégalité des races). Le tableau suivant présente le relevé des segments les plus fréquents contenant un modificateur à fonction classificatrice; il établit ainsi une typologie des inégalités les plus citées en 2015 :

\begin{tabular}{|l|l|}
\hline Segments & Fréquences \\
\hline
\end{tabular}




\begin{tabular}{|l|l|}
\hline inégalités sociales & 138 \\
\hline inégalités de revenu(s) & 37 \\
\hline inégalités scolaires & 23 \\
\hline inégalité de traitement & 21 \\
\hline inégalités territoriales & 20 \\
\hline inégalité des races & 17 \\
\hline inégalités économiques & 16 \\
\hline inégalités salariales & 13 \\
\hline inégalité sociale & 13 \\
\hline inégalités de santé & 13 \\
\hline inégalités de salaires & 12 \\
\hline
\end{tabular}

TABLEAU 1. Inégalité(s) + modificateur (segments répétés ${ }^{6}$, Lexico3)

La variété des expressions relevées par le logiciel montre que le nom inégalité(s) se caractérise, dans le discours de presse, par une grande diversité référentielle ${ }^{7}$ avec une prédominance pour le syntagme inégalités sociales -, chaque segment renvoyant à un référent spécifique. Dans les énoncés (6) et (7), on voit que le même mot recouvre des entités distinctes : l'adjectif relationnel sociales, en regroupant les inégalités autour de cette propriété, établit une nouvelle "classe référentielle " (Bosredon $1988: 4$ ), une sous-catégorie d'inégalités parmi d'autres. En bref, le champ référentiel d'inégalité(s), dans le corpus, englobe un ensemble divers de référents qui se distinguent fondamentalement suivant le domaine dans lequel le nom est rattaché: social, économique, culturel, scolaire (8).

(6) Les études mesurant les gains économiques des politiques de lutte contre les inégalités sont un piège, nous rappelle l'excellent programme de recherche Presage de Sciences-Po. (Libération, 01-12-2015)

(7) [L]es mots d'ordre [du Mouvement du 20-Février] - dignité, liberté et lutte contre les inégalités sociales - continuent à être portés. (Le Monde, 03-09-2015)

(8) Qu'est-ce qu'une République qui, aux inégalités sociales, économiques, culturelles, vient ajouter chaque jour de nouvelles inégalités scolaires? (Libération, 26-02-2015)

11 Cela dit, l'emploi d'un adjectif relationnel, même s'il apporte une valeur référentielle spécifique à inégalité(s), ne suffit pas à dissiper les interrogations concernant l'interprétation du nom, dans la mesure où les expressions visées renvoient rarement à des entités identifiables. En effet, dans les extraits (7) et (8), on n'arrive pas à déterminer si le référent désigné par le syntagme nominal concerne des inégalités de la société ou dans la société : la société est-elle la source, ou bien le siège des inégalités ? On relève, en outre, l'absence d'informations plus précises sur les entités jugées inégales : si le mot au pluriel tend, certes, à désigner des faits concrets (cf. infra $§ 3.1 .1$ ), il est, 
toutefois, difficile, dans les cotextes retranscrits, d'isoler, d'individualiser, les situations, qui dans la société, sont désignées par le segment inégalités sociales.

12 En mettant en œuvre une conception moins référentielle de la signification, les définitions des dictionnaires permettent souvent de repérer les valeurs qui s'accrochent à un mot de façon stable et partagée. On verra maintenant les traits sémantiques mis en avant par les rédacteurs du Trésor de la langue française (TLF) et du Petit Robert dans le cas d'inégalité(s).

\subsection{Une définition de dictionnaire détachée de l'histoire}

On observe d'abord que les dictionnaires font appel à des critères morphologiques pour établir la définition d'inégalité(s). En effet, le TLF s'appuie sur l'origine adjectivale du nom pour le définir comme le "fait d'être inégal », tandis que Le Petit Robert met en avant l'une des valeurs du préfixe in-, l'inégalité étant considérée comme une privation, un «défaut» d'égalité ou de régularité. Ensuite, à partir de l'analyse de la complémentation du nom, les lexicographes distinguent deux acceptions d'inégalité. Dans la première, le terme est défini comme le fait de présenter une ou des différences quantitatives ou qualitatives; le constat d'inégalité implique, ici la comparaison entre des entités (personnes ou choses) qui, n'étant pas de même nature, quantité, qualité ou valeur, sont alors perçues comme différentes, inégales (inégalité entre les hommes et les femmes, entre les états). Dans la seconde, le mot décrit le fait de "présenter des différences dans son aspect ou dans son développement " (TLF) ; elle concerne une entité prise isolément, laquelle présente des irrégularités en elle-même (inégalité de surface) ou manifeste un caractère variable (inégalités d'humeur) ${ }^{8}$.

Mais le sens d'inégalité(s) dans le discours sociopolitique va au-delà de ces quelques éléments de définition fournis par les dictionnaires de langue. Depuis le xvIII ${ }^{\mathrm{e}}$ siècle, période qui marque l'entrée du mot égalité dans le lexique politique (Dubois 1962 : 78), et à la suite du Discours sur l'origine et les fondements de l'inégalité parmi les hommes, de J.-J. Rousseau - essai dans lequel l'auteur interroge la cause réelle de l'inégalité -, des valeurs et des représentations, se sont, au fil du temps et des événements, rattachées à la forme étudiée. Ainsi, le plaidoyer de Babeuf en faveur de l'égalité de fait et de la propriété collective annonçait, déjà en 1795 , les revendications des communards contre l'inégalité, laquelle, étant associée à l'idée de privilège, apparaît, dans le discours contestataire, comme source de révoltes: «Les causes de toutes les révolutions sont: les privilèges, l'inégalité devant les résultats, entre le capital et le travail », prônaient les insurgés en $1871^{9}$. Avec la Révolution française, les mots égalité et inégalité se sont donc constitués en lieux de conflictualité - les partisans de l'égalité se sont heurtés à la défense vigoureuse de l'ordre établi inégalitaire, celui de l'Ancien Régime - et sont depuis devenus des « supports » de revendications politiques et sociales :

Fondements immuables du discours républicain, ils [les termes égalité et inégalité(s)] sont aussi les supports dynamiques de revendications démocratiques et de luttes sociales pour des droits qui ont été progressivement définis, conquis, mais aussi périodiquement remis en cause : parité entre les sexes, justice sociale, droit à la santé, à l'instruction, au travail, au logement. (Fiala 1999: 7)

15 On le voit, les traits sémantiques entrant dans la composition des définitions de dictionnaire, à savoir [+ absence/négation d'égalité], [+ différence] et [+ comparaison], 
ne tiennent pas compte des valeurs qui se sont communément associées au mot au cours de l'histoire politique et sociale contemporaine. Or, l'analyse du corpus de presse montrera que l'usage et le sens d'inégalité(s) est en lien étroit avec l'expérience humaine, avec des représentations et des pratiques sociales historicisées, même si l'on peut déjà, semble-t-il, entrevoir des traces de cette association dans l'idée de comparaison présente dans les définitions : l'inégalité supposant la mise en parallèle d'entités distinctes, c'est donc bien l'expérience provenant d'un acte d'observation qui permet d'établir le fait d'être inégal et d'attribuer, à ce fait, le nom d'inégalité.

\section{Pour une approche discursive du sens}

Ces interrogations préliminaires suscitent trois remarques. En premier lieu, dans la mesure où il est difficile de cerner le référent d'inégalité(s) en discours en raison de son fonctionnement variable - selon les emplois, le référent fluctue entre fait abstrait ou manifestation concrète de ce fait et se décline selon le domaine dans lequel le mot apparaît -, l'analyse sémantique du nom ne peut donc pas reposer sur la description de ses propriétés référentielles. En deuxième lieu, le recours aux dictionnaires, qui généralement fournissent quelques informations utiles pour «se faire une idée du sens d'un mot " (Cusin-Berche 1998 : 150), s'avère, dans le cas d'inégalité(s), peu productif, puisque les définitions, qui sont assez courtes, ne tiennent pas suffisamment compte des usages sociaux et politiques du nom, dans lesquels l'inégalité est en lien avec l'idée de privilège. Enfin, on constate que, malgré cette instabilité référentielle et ces imprécisions définitionnelles, le mot apparaît de façon courante dans le discours de la presse - comme le signale le nombre important d'occurrences relevées pour la seule année 2015 -, ce qui amène donc à penser qu'il n'engendre pas de difficulté d'emploi et de compréhension majeure.

Ces observations nous conduisent à aborder le fonctionnement sémantique d'inégalité(s) par le biais d'une approche qui, à la différence des perspectives référentielle et différentielle, plaide pour la prise en compte, dans l'analyse, de la dimension discursive du sens : dans cette optique, le référent d'une unité lexicale n'est plus considéré comme un donné, existant préalablement à la production discursive et étant disponible en dehors du langage ; il est plutôt envisagé comme le résultat d'une "reconstruction » effectuée par les locuteurs et saisie à travers le discours (cf. Moirand 2011). Cette position implique donc de concevoir le sens, non pas comme le produit de la description d'un référent unique, mais plutôt comme une entité essentiellement composite, une combinaison des principales dimensions dans lesquelles le mot est utilisé. Ces dimensions, qui ressortent de l'analyse du cotexte (l'intradiscours), sont multiples et définies par le corpus; elles reflètent les différents usages discursifs d'un mot dans un corpus délimité et sont à mettre en lien avec des pratiques et des représentations, avec le contexte dans lequel les discours sont produits. Pour reprendre les termes de S. Moirand, on peut enfin dire que le sens constitue, dans cette approche, « un lieu de convergence entre la langue, le discours et la culture (à travers la mémoire et l'histoire) » $(2011: 176)$.

18 Sur le plan méthodologique, la démarche entreprise consiste, plus précisément, à établir le sens d'inégalité(s) à partir des constructions dans lesquelles le mot se trouve et des associations avec d'autres termes, car, comme le dit M. Tournier (1996: 76), « un mot n'existe pas par lui-même ; il est lu et entendu à travers les mots qui ont l'habitude 
de coexister avec lui dans le discours ». Ainsi, en nous appuyant sur l'idée selon laquelle le cotexte (étroit et élargi) d'un mot entre dans la composition de son sens ${ }^{10}$, nous chercherons à établir les dimensions sémantiques du nom à partir de ses usages en discours les plus récurrents et les plus saillants. En bref, c'est autour du cotexte envisagé comme un moyen d'aborder le mot par des phénomènes relevant de plusieurs niveaux d'analyse linguistique (syntaxique, lexical, mais aussi énonciatif et pragmatique) - que l'étude sémantique et discursive d'inégalité(s) s'organisera.

Notre proposition se distingue, par ailleurs, des travaux qui cherchent à étudier le sens des noms à travers des propriétés formelles marquées linguistiquement, même si nous y avons souvent recours pour comprendre les fonctionnements discursifs (cf. § 3.1.1, infra). De fait, la méthode de la "sémantique linguistique", qui vise à établir des "propriétés sémantiques que la langue choisit de marquer formellement dans ses structures" (Flaux et Van de Velde 2000: 2), repose sur des données non contextualisées, contrairement à notre projet, qui place les données issues de corpus au cœur de son dispositif. La présente étude n'a donc pas pour objectif d'aborder la question sémantique en cherchant à classer le nom inégalité(s) selon ses propriétés syntaxiques (a-t-on affaire à un nom d'état, de sentiment ou de qualité ?), mais elle s'attache à la travailler en discours, à partir des usages les plus récurrents du mot dans des textes de la presse française.

Ainsi, par la place qu'elle consacre au co(n)texte dans l'analyse du sens, la recherche que nous présentons dans ce qui suit s'ancre finalement dans le projet de constitution d'une "sémantique discursive", en ce qu'elle cherche à rendre compte de la construction du sens en et par le discours (Guérin, Lecolle \& Veniard 2018 : 6), à partir d'un corpus délimité. Certes, la démarche sémasiologique appliquée ne permet pas de capter d'autres actualisations linguistiques associées à la question de l'inégalité (comme discrimination, exclusion, fracture sociale, etc.), mais elle rend possible le repérage, grâce aux logiciels Lexico3 et Le Trameur, des fonctionnements les plus fréquents du mot dans le corpus et, par conséquent, la mise au jour des dimensions sémantiques d'inégalité(s).

\section{Dimensions sémantiques d'inégalité(s)}

21 L'étude propose une articulation entre le qualitatif et le quantitatif: des données quantitatives, obtenues grâce aux logiciels de statistique textuelle, viendront appuyer, en amont ou en aval, l'analyse de la forme inégalité(s) dans ses cotextes et dans le corpus de presse rassemblé. La mise en œuvre de cette méthode a permis d'établir le sens d'inégalité(s) en discours, lequel se déploie en trois dimensions principales.

\subsection{L'inégalité(s) en tant que lieu d'observation}

Nous rassemblons ici les usages récurrents d'inégalité(s) dans lesquels le mot est présenté dans sa dimension empirique, comme renvoyant à un fait pluriel, concret et mesurable. L'inégalité fonctionne, dans ces constructions, comme fait ancré dans le réel, dans la vie d'une société, qui peut être observé, décrit et appréhendé au moyen notamment de données chiffrées. 


\subsubsection{Un fait pluriel et concret} déséquilibrée, dans le corpus, entre le singulier et le pluriel de la forme. De fait, inégalité(s) apparaît essentiellement au pluriel : 1439 occurrences du mot au pluriel ont été relevées - soit $82,4 \%$ de l'ensemble des occurrences d'inégalité(s) -, contre seulement 307 occurrences du mot au singulier. La mise au jour de cet emploi préférentiel est significative, car l'opposition entre le singulier et le pluriel s'accompagne souvent d'une différence sur le plan sémantique, liée notamment à l'opposition entre l'abstrait et le concret ${ }^{11}$, et cela en particulier dans le cas - très représentatif dans le corpus ${ }^{12}$ - de référence générique (cf. Gary-Prieur 2011 : 39-43).

Comme nous l'avons montré (cf. supra $\$ 1.1$ ), l'usage d'inégalité(s) en discours s'accompagne d'une certaine indétermination référentielle, le nom pouvant, selon les cotextes, renvoyer à une entité abstraite (le thème, le sentiment d'inégalité), ou plutôt à la manifestation concrète du fait inégal (une situation d'inégalité). L'extrait qui suit illustre cette instabilité à travers l'opposition entre le singulier et le pluriel, mise en relief dans le même énoncé :

(9) Opposant les sociétés démocratiques aux temps aristocratiques, Tocqueville écrivait ainsi dans De la démocratie en Amérique: «Quand l'inégalité est la loi commune d'une société, les plus fortes inégalités ne frappent point l'œil ; quand tout est à peu près de niveau, les moindres le blessent. C'est pour cela que le désir de l'égalité devient toujours plus insatiable à mesure que l'égalité est plus grande. » (Le Monde, 11-03-2015)

Dans cet exemple, le mot au singulier renvoie à l'inégalité dans sa généralité abstraite, en tant que catégorie, qui, par ses propriétés, s'oppose à toutes les autres catégories, comme à celle de l'égalité. L'usage du générique intensionnel (Martin 1996: 50) sert ainsi à accroître le degré d'abstraction: dans l'inégalité, le nom est appréhendé en intension, à partir des propriétés qui permettent de le définir comme un concept. L'abstraction est aussi renforcée par le parallélisme avec la loi (substantif qui fait référence à une entité immatérielle), l'inégalité pouvant s'ériger, comme c'est le cas dans les sociétés aristocratiques, en « loi commune d'une société ", selon la formulation de Tocqueville. Le fait d'être inégal devient alors le trait qui définit une société.

L'inégalité en tant que concept, «loi », diffère, des inégalités qui " ne frappent point l'œil » ou qui «le blessent». Le pluriel semble, dans ce cas, s'orienter vers plus de concrétude, dans la mesure où l'inégalité est appréhendée à travers ses manifestations sensibles, perceptibles. Le générique extensionnel sert d'ailleurs à renforcer le passage vers le concret: dans les inégalités, le défini pluriel conduit à interpréter le nom en extension, en visant la totalité des situations qui entrent dans la composition de la catégorie de l'inégalité. La présence du pluriel entraîne ainsi un déplacement au niveau sémantique : la focalisation passe de l'inégalité, perçue comme une entité unifiée et définie par une propriété, le fait d'être inégal (plus abstrait), aux situations, multiples et diverses, qui possèdent cette propriété (plus concret).

L'examen de cet extrait nous conduit ainsi à avancer l'idée selon laquelle, dans les discours de presse, l'usage de l'article défini au pluriel fonctionne comme une procédure de concrétisation (Martin 1996), faisant pencher le nom du côté du concret. Ce constat peut d'ailleurs expliquer la surreprésentation de la forme plurielle dans le corpus : le rôle de la presse étant avant tout de porter à la connaissance de ses lecteurs des événements, ou des situations, ayant une importance pour la société, l'usage du 
pluriel semble donc « aller de pair » avec cette dimension empirique, concrète, centrale dans la pratique des journalistes. À titre de comparaison, une vérification sur Frantext (ATILF-CNRS) montre la prédominance du singulier dans un corpus qui, quant à lui, est composé essentiellement de textes littéraires et philosophiques; nous avons relevé un total de 2388 occurrences d'inégalité, contre 902 occurrences d'inégalités, ce qui représente une tendance inverse par rapport au corpus de presse ${ }^{13}$ :

Figure 1 : Fréquences d'inégalité(s) dans les corpus de presse et Frantext (Lexico3)

Ainsi, la comparaison avec le corpus issu de la base Frantext, dans laquelle l'usage de la presse est très peu représenté, conduit à penser que l'emploi de la forme au pluriel constitue une routine dans la sphère journalistique. En effet, moins propice au débat philosophique et conceptuel sur l'inégalité (sauf dans certaines rubriques spécifiques), la presse privilégie, tout au long de ses textes, les situations multiples et variées d'inégalité, lesquelles font l'objet de revendications de groupes sociaux divers (cf. infra $\S 3.3$ ), dont les paroles et les positions, parfois antagoniques, sont rapportées et rendues visibles par les médias.

\subsubsection{Un fait mesurable}

Lorsque l'on étudie de plus près le cotexte d'inégalité(s), on relève également des constructions dans lesquelles le mot apparaît comme un fait empirique mesurable ${ }^{14}$, qui est susceptible d'être quantifié et défini en termes d'intensité. L'expression de cette dimension passe, dans le corpus, par l'usage fréquent de données chiffrées d'une part et par la présence, dans l'entourage proche d'inégalité(s), de mots dénotant l'intensité d'autre part.

Le recours à des chiffres et des statistiques est ainsi l'un des procédés utilisés pour exprimer la dimension mesurable du mot inégalité(s). On voit bien, en (10), l'emploi de données chiffrées pour décrire quantitativement et, par conséquent, "objectiviser " (Moirand 2016) le fait inégal. Souvent produites par des institutions et des économistes considérés comme « faisant autorité » en la matière - par exemple, l'OCDE (10), Thomas Piketty (11), les Nations unies (12) -, les chiffres donnent à une réalité sociale une représentation mathématique intelligible: l'inégalité n'est donc plus une abstraction, elle devient un objet que l'on compte, qui peut être quantifié et qui peut donner lieu à des comparaisons (aujourd'hui/dans les années 80, entre 1958 et 2005, entre 1990 et 2010). Mise en chiffres, l'inégalité devient ainsi un fait objectif et universel, car, comme l'affirment P. Bacot, D. Desmarchelier et S. Rémi-Giraud, «[1]e monde des chiffres se présente comme celui de l'objectivité, mais aussi comme celui de l'universalité: objectivité résultant du protocole gouvernant leur production; universalité garantie par un système de signes translinguistiques et, partant, transculturels » (2012:11).

(10) De quoi appuyer l'idée que la concentration des richesses ne fait qu'accentuer des inégalités dévastatrices. En mai, une étude de l'OCDE reconnaissait qu'elles n'avaient «jamais été aussi élevées» dans les pays développés. Où le revenu des $10 \%$ les plus riches est 9,6 fois plus élevé aujourd'hui que celui des $10 \%$ les plus pauvres (contre 7 fois plus dans les années 80). «La montée des inégalités, entre 1985 et 2005, dans les 19 pays de l'OCDE analysés, a amputé la croissance de 4,7 points de pourcentage en cumulé entre 1990 et 2010 ", notait l'OCDE. (Libération, 17-09-2015)

(11) Jeudi, l'économiste français Thomas Piketty, chroniqueur à Libération et dont Le Capital au XXI siècle s'est écoulé à 1,5 million d'exemplaires, s'imposant dans de nombreux pays comme un ouvrage de référence sur le processus d'accroissement des inégalités [...]. (Libération, 02-01-2015) 
Cela dit, dans le corpus, l'expression du caractère mesurable d'inégalité(s) ne se limite pas aux données chiffrées; on trouve également des indices de cette dimension dans des constructions où le mot est caractérisé par des verbes, des noms verbaux ou des nominalisations qui expriment l'idée de montée ${ }^{15}$ des inégalités : en effet, à partir du relevé des segments répétés, on note que le mot apparaît, avant tout, comme un phénomène intense, qui est en augmentation (creusement, accroissement, hausse, se creusent, aggravation) :

\begin{tabular}{|l|l|}
\hline Segments & Fréquences \\
\hline creusement des inégalités & 29 \\
\hline montée des inégalités & 28 \\
\hline accroissement des inégalités & 22 \\
\hline augmentation des inégalités & 20 \\
\hline hausse des inégalités & 19 \\
\hline inégalités se creusent & 15 \\
\hline aggravation des inégalités & 13 \\
\hline explosion des inégalités & 10 \\
\hline fortes inégalités & 10 \\
\hline
\end{tabular}

TABLEAU 2. Un fait intense et en augmentation (segments répétés, Lexico3)

Le mot est, en outre, modalisé par des adjectifs (fortes), des expressions adverbiales (fortement, encore plus) et des expressions verbales qui impliquent un poids mesurable économiquement (grèvent) ou une scalarité (ne cessent de) :

(12) Le rapport des Nations unies sur l'IDH pointe les fortes inégalités qui grèvent le développement en Inde, et qui feraient perdre au pays près du tiers de son IDH. Elles sont d'abord scolaires. Seuls $42 \%$ des jeunes de moins de 25 ans ont poursuivi leurs études jusqu'au secondaire. (Le Monde, 22-12-2015)

(13) La présidente du groupe républicain au conseil régional d'île-de-France a rappelé les « deux urgences » majeures qui l'ont conduite à un accord de principe avec les centristes de l'UDI. "Le social, tandis que les inégalités ne cessent de s'accroître dans notre région. Et l'écologique pour arrêter les dégâts sur la santé de nos habitants », a insisté la députée des Yvelines [...]. (Le Figaro, 19-08-2015)

(14) Aux États-Unis, les inégalités de revenu ont fortement augmenté depuis les années 70, comme l'a montré notamment le travail de Thomas Piketty. Cette montée des inégalités aurait pu amener les Américains à devenir plus favorables aux politiques de redistribution du revenu. Or, il n'en est rien. (Libération, 14-04-2015)

(15) Mais ces inégalités s'aggravent encore plus outre-Atlantique !

Oui, mais pas parce qu'ils échouent dans leur transition numérique. Il leur manque les institutions pour mieux répartir la colossale richesse qu'elle crée et assurer les individus. C'est un fait que cette mutation accroît les inégalités et exerce des effets très négatifs sur les classes moyennes. (Libération, 09-11-2015) 
Enfin, la présence fréquente de déverbaux et de nominalisations dans l'entourage d'inégalité(s) - ces mots apparaissent en tête de liste des segments répétés (cf. tableau 2, supra) - nous conduit à interroger le fonctionnement de ces constructions. Si, dans certains exemples, comme en (14), le syntagme Cette montée des inégalités reprend anaphoriquement la prédication énoncée dans la première phrase, les inégalités de revenu ont fortement augmenté, cette transition d'un fait qui s'appuie sur des statistiques, sur des résultats d'enquêtes de spécialistes (comme l'a montré notamment le travail de Thomas Piketty), vers une donnée désormais acquise n'est souvent pas explicitée. De fait, on trouve, dans le corpus, des extraits, comme en (16), où la «démonstration statistique » de la dite montée n'est pas apportée. Or, en absence d'assertion antérieure, on peut interpréter les nominalisations et les déverbaux comme des traces d'un discours antérieur, déjà dit, déjà connu et déjà accepté ${ }^{16}$. Et c'est ainsi que l'on interprète le fonctionnement du segment la montée des inégalités en (16) : la trajectoire vers le haut des inégalités étant une information acquise, confirmée par des études d'experts, elle n'a donc pas toujours besoin d'être démontrée et peut d'ailleurs, sous le mode de l'évidence, intégrer l'argumentation politique (la montée des inégalités, l'un des deux grands défis de notre époque). À partir de l'analyse de ces segments, on peut avancer l'idée selon laquelle, dans ces constructions, l'accent n'est finalement pas mis sur le nom inégalité(s), lequel se trouve en position effacée (en fonction de complément), mais plutôt sur l'événement (la montée) : c'est bien la hausse des inégalités qui est visée en (16), et non pas les inégalités en elles-mêmes.

(16) Le travail dominical, que prévoit d'assouplir le texte, est une "véritable régression » et une "menace pour la cohésion sociale », estime l'écologiste [C. Duflot] en plaidant pour « un nouveau modèle social écologique, capable de répondre aux deux grands défis de notre époque : le dérèglement climatique et la montée des inégalités ». (Le Figaro, 05-01-2015)

L'inégalité comme fait pluriel, concret et mesurable ne constitue qu'une dimension sémantique du nom. La connotation négative dont peut se charger inégalité(s), comme on le verra ci-après, apparait comme la condition nécessaire pour que le mot devienne support de revendications $(\$ 3.3$, infra).

\subsection{L'inégalité(s) en tant que lieu d'évaluation}

Dans le corpus de presse, le mot peut être évalué de façon négative et apparaître comme un fait injuste, dont l'existence porte préjudice à la société. Dans les sociétés contemporaines, qui sont marquées par l'égalité de droits, les inégalités sont de moins en moins tolérées, car elles revoient à l'idée de privilège contre laquelle des individus se sont dressés ${ }^{17}$. Plusieurs usages en discours illustrent cette dimension d'inégalité(s).

Comme nous l'avons exposé plus haut (cf. $§ 1.3$ ), la comparaison est un élément essentiel dans la définition lexicographique du terme inégalité. Cela dit, dans inégalité de deux hauteurs, exemple canonique que l'on trouve souvent dans les dictionnaires, le nom n'est pas chargé de connotation; il dénote uniquement le fait que les deux hauteurs comparées présentent des différences quantitatives et qu'elles sont, de ce fait, inégales. Ici, la comparaison entre les entités ne donne lieu à aucun questionnement sur la nature de l'inégalité observée. Il en va autrement de l'énoncé qui pose l'inégalité entre les hommes, dans la mesure où la comparaison entre les statuts des membres d'une société - et le constat de différence qui en découle - peut conduire à questionner le caractère juste, ou non, de l'inégalité. Quand la comparaison donne lieu à l'évaluation 
des entités mises en parallèle, le constat de différence qui s'ensuit peut donc devenir source d'injustice. L'examen du corpus de presse montre d'ailleurs une proximité entre les deux mots, qui peuvent, en discours, entretenir des relations de reprise, comme dans les extraits suivants :

(17) Ils [les pauvres] nous rappellent les inégalités, les discriminations, le déni de reconnaissance, auxquels nous participons malgré nous par passivité. Les plus chanceux racontent aussi comment la prise de parole et les actions menées dans des collectifs leur ouvrent la voie vers la dénonciation de ces injustices. (Libération, 26-05-2015)

(18) Or Marisol Touraine déplore une inégalité dans l'accès à ces soins [palliatifs]. "Cette injustice, tant sociale que territoriale, est inacceptable », déclare-t-elle. (Le Figaro, 12-10-2015)

Plus précisément, ces exemples illustrent la présence du sème [- positif] dans le cotexte proche d'inégalité(s), notamment dans les unités dénonciation (17), déplore et inacceptable (18). Mais, dans le corpus, les usages qui actualisent ce trait négatif peuvent être divers, et l'on trouve, dans les associations avec malade (19) et poison (20), l'expression de l'idée selon laquelle l'inégalité est un problème (21), une "situation instable ou dangereuse » (Le Petit Robert), qui peut être à l'origine de troubles sociaux, de déséquilibres (22), et qu'il faut résoudre (21).

(19) «Le parlement ne peut plus se permettre de renvoyer le texte de loi [concernant le contrat d'union civil en Italie], a estimé $\mathrm{M}^{\mathrm{me}}$ Boldrini. Nous ne pouvons plus être un pays malade d'inégalités sociales et économiques. » (Le Monde, 23-07-15)

(20) Transformer le poison de l'inégalité, de la frustration, de la haine - celle qui prend parfois un grand « $\mathrm{H} »-$ en une proposition de projet que personne ne peut refuser, voilà le moteur qui vient de permettre aux jeunes du Bondy Blog de faire des miracles [...]. (Libération, 26-10-2015)

(21) Cette formule d'«apartheid» est insupportable. Depuis trente ans, on a cherché à résoudre les problèmes de discrimination et d'inégalité en accumulant des réglementations et c'est un échec. (Le Figaro, 14-11-2015)

Par ailleurs, le Brésil souffre de profonds déséquilibres : [...] l'indigence des services publics, particulièrement dans l'éducation et la santé; l'ampleur des inégalités sociales et territoriales; la faiblesse de l'État de droit, qui favorise une corruption endémique et la montée de la violence. (Le Figaro, 09-02-2015)

L'observation des cooccurrents ${ }^{18}$ fait également ressortir la présence de ce trait. En effet, parmi les mots qui côtoient régulièrement inégalité(s) dans son environnement phrastique, plusieurs renforcent cette dimension négative ; c'est le cas, par exemple, de l'adjectif insupportables, qui comporte le sème [- positif] dans le préfixe in-, mais aussi du verbe corriger, qui exprime l'idée d'un redressement - «Ramener à la règle (ce qui s'en écarte) » (Le Petit Robert) - et demande souvent, en complément, un élément jugé négatif : corriger les fautes, les erreurs, les défauts et donc aussi les inégalités.

\begin{tabular}{|l|l|l|}
\hline Pôle & Cooccurrent & Spécificité \\
\hline inégalité & injustice & 10 \\
\hline \multirow{2}{*}{ inégalités } & contre & 46 \\
\cline { 2 - 3 } & criantes & 28 \\
\cline { 2 - 3 } & aggravation & 17 \\
\cline { 2 - 3 } &
\end{tabular}




\begin{tabular}{|l|l|}
\hline corriger & 15 \\
\hline injustice(s) & 8 \\
\hline aggraver/aggravent & 8 \\
\hline insupportables & 7 \\
\hline
\end{tabular}

TABLEAU 3. Un fait jugé négativement (extrait des cooccurrents d'inégalité(s), Le Trameur)

On relève, en outre, l'association privilégiée entre inégalités et la préposition contre, qui peut exprimer une relation d'opposition et d'adversité: on comptabilise 103 occurrences du segment contre les inégalités, la plupart en complément des formes lutte et lutter (75 occurrences). Dans l'extrait qui suit, par exemple, on voit que la préposition contre met en relation deux pôles - le pape François et les inégalités - qui se trouvent dans une relation de tension, le premier visant à la réduction ou à la suppression du second ${ }^{19}$, placé en adversaire.

(23) Deux grands groupes, l'un libéral, l'autre conservateur, structurent le monde catholique américain, explique l'intellectuel. [...] Ce groupe [libéral, en ligne avec le Parti démocrate] est donc particulièrement sensible au combat contre les inégalités mené par le pape François. (Le Figaro, 23-09-2015)

On peut, certes, trouver des énoncés où c'est plutôt le « degré de l'inégalité » qui pose question (24), reprenant le discours selon lequel l'efficacité économique dépend de la liberté individuelle et exige, au moins, une dose d'inégalité. Cela dit, le recours, dans cet extrait, à un marqueur de modalité (peut) signale la difficulté qu'il y a aujourd'hui à tenir un discours manifeste en faveur de l'inégalité ${ }^{20}$ :

(24) [L]es auteurs de ce travail, qui lance le débat au FMI sans engager pour l'instant l'institution, prennent soin de faire remarquer qu'un "certain degré d'inégalité peut ne pas être un problème dans la mesure où cela incite les individus à exceller, à se battre, à épargner et à investir pour aller de l'avant ». (Le Monde, 17-06-2015)

41

Les exemples soulignent que la forme inégalité(s) apparaît, dans le corpus, associée notamment à des noms, des verbes et des adjectifs porteurs d'un jugement de valeur essentiellement négatif. Dans ces cotextes, la focalisation se fait donc sur la dimension négative, injuste du mot; en tant qu'injustice, en tant que problème, l'inégalité pose question et appelle une réponse, une praxis: la lutte contre et pour la réduction des inégalités.

\subsection{L'inégalité(s) en tant que lieu de revendications}

Nous rassemblons ici les usages les plus fréquents d'inégalité(s) dans lesquels le mot apparaît comme lieu de revendications politiques et sociales: une fois observée et mesurée, l'inégalité est souvent jugée négativement; elle devient alors un fait qui doit être combattu et réduit, même si parfois ce combat semble revêtir un caractère très « convenu ».

\subsubsection{Un fait contre lequel il faut lutter et qu'il faut réduire}

L'étude des verbes et des noms verbaux qui accompagnent inégalité(s) a montré que l'usage de ce mot, dans le corpus, est lié à l'idée d'augmentation: creusement, montée, 
accroissement des inégalités (cf. § 3.1.2, supra). Mais le mot peut également se trouver en association avec l'idée de diminution : ainsi, le relevé des cooccurrences atteste une attraction forte et une cohabitation régulière, à l'échelle de la phrase, entre inégalités et les formes réduire (**, spécificité $>50$ ) et réduction (spécificité : 38 ); même si le trait [+ diminution] n'est pas intrinsèquement inhérent aux mots lutte (**, spécificité $>50)$ et lutter (spécificité : 30), on peut déduire de la combinaison avec inégalités que l'action de lutter contre a pour objectif de diminuer les inégalités.

Si l'on aborde le mot d'un point de vue énonciatif, on observe que cette tendance à la diminution, notamment en ce qui concerne le segment lutte(r) contre les inégalités, apparaît souvent dans des genres discursifs où la présence de l'énonciateur est davantage marquée, comme des articles d'opinion, des analyses, des tribunes et des interviews. Lorsque la focalisation se fait sur la dimension négative, injuste du mot (cf. $\S 3.2$ ), un positionnement neutre face au phénomène paraît difficile, et l'énonciateur peut alors laisser davantage de traces de subjectivité dans ses dires (Kerbrat-Orecchioni 2009) ; on trouve ainsi, dans le cotexte proche d'inégalité(s), des marques de l'ironie, une ponctuation expressive - Merci pour le compliment! (25) -, et de multiples modalités appréciatives : triste record (26), égalité bafouée, fuite en avant catastrophique (27).

(25) Les études mesurant les gains économiques des politiques de lutte contre les inégalités sont un piège, nous rappelle l'excellent programme de recherche Présage de Sciences-Po. [...]

Un autre argument classique pour justifier l'intégration des femmes est qu'elles ont un autre regard, sont moins conflictuelles, plus rigoureuses, bref elles sont complémentaires aux hommes. Merci pour le compliment ! (Libération, 01-12-2015, chronique)

(26) Triste record, nous avons choisi le thème le plus emblématique, celui de la jeunesse. En effet, plus de 800000 demandeurs d'emploi, toutes catégories confondues, ont moins de 25 ans : un record ! Près de 2 millions de jeunes sont sans diplôme et sans emploi. La question de leur insertion sur le marché du travail est primordiale. Pour la cohésion de la société française, nous avons plus que jamais une obligation de résultats. Résultats dans la lutte contre les inégalités scolaires et dans l'enseignement supérieur, contre le décrochage, contre les discriminations encore si marquées, contre les inégalités dans l'accès à la santé, contre le chômage de masse... trop souvent, la République ne tient plus ses promesses élémentaires, trop souvent ses responsables sont pris en flagrant délit de déni, voire de cynisme. (Le Monde, 24-11-2015, tribune)

(27) «L'égalité d'accès aux savoirs est bafouée "

[Journaliste] Cette réforme prétend lutter contre les inégalités. Qu'en pensezvous?

[Charles Coutel, philosophe et professeur à l'université d'Artois] Au lieu de tirer les leçons de l'échec du collège unique, le ministère actuel décide d'une fuite en avant catastrophique, dictée à évidence par le calendrier électoral. (Le Figaro, 10-10-2015, interview)

À part un exemple dans lequel l'énonciateur insiste sur le constat qu'il y a effectivement lutte contre et réduction des inégalités en France (article paru dans Libération le 22-09-2015), la plupart des extraits révèle des actes de parole que l'on peut classer en trois catégories selon la position sociale ou politique de l'énonciateur ${ }^{21}:$ des actes de critique envers l'inefficacité de la lutte menée contre les inégalités; des actes de promesse où les énonciateurs affichent l'intention de lutter; et des actes de revendication de lutte contre les inégalités.

Dans le premier cas de figure, les énonciateurs remettent, par exemple, en cause les gains d'une lutte présupposée existante (effet d'évidence engendré par le syntagme 
nominal lutte contre les inégalités), considérée comme inefficace et insuffisante, comme le suggèrent le présupposé de la modalité aléthique une vraie lutte en (28) ainsi que le verbe améliorer en (29). La lutte est aussi qualifiée d'échec (30) et caractérisée comme une promesse non tenue en (26) et (31).

(28) Nous ne pouvons pas repartir comme avant. J'ai entendu Manuel Valls parler de «l'esprit du 11 janvier ». Il a raison. Mais cela oblige à agir, à mener une politique différente qui réponde à cet "esprit du 11 janvier » : plus de fraternité et de services publics, une vraie lutte contre les inégalités et la relégation. (Libération, 29-01-2015, interview)

(29) Médecins du monde, ainsi que d'autres associations vont lancer un appel aux parlementaires "pour améliorer ce projet de loi santé en matière de lutte contre les inégalités ». (Libération, 25-02-2015)

(30) Pour le premier ministre, qui ne s'était pas exprimé dimanche soir, pas question de reconnaître que l'échec de la gauche, au pouvoir depuis 2012, sur le front du chômage et de la lutte contre les inégalités est en partie responsable de la montée du FN, notamment dans les milieux populaires. (Le Monde, 09-12-2015)

(31) Une multitude d'autres pistes sont à creuser, touchant aux modes de gouvernance (en France, on a encore du chemin à faire pour coconstruire l'intérêt général) [...], et plus généralement à la lutte contre les inégalités sociales, culturelles, raciales, de sexe, économiques - inégalités qui constituent le terreau du silence politique.

Oui, le cercle des injustices et du silence politique est vicieux. Oui, les promesses non tenues à propos du récépissé de contrôle d'identité, du droit de vote des étrangers, de la lutte contre les inégalités sociales alimentent ce cercle vicieux. (Libération, 26-05-15, tribune)

Dans le deuxième cas de figure, on relève des exemples de «bonnes intentions » en termes de lutte contre les inégalités, intentions que l'on pourrait aussi interpréter comme des promesses, si l'on considère que, lorsqu'une femme ou un homme politique s'exprime, sa visée est aussi de convaincre des électeurs avec des annonces prometteuses. Les noms défi, objectifs et les verbes vouloir et plaider côtoient alors l'action politique annoncée en (32), (33) et (34), à savoir celle de réduire les inégalités. Ces actions sont tantôt des annonces des énonciateurs eux-mêmes (32-33), tantôt des interprétations effectuées par les journalistes à propos de l'acte de parole de l'énonciateur dont ils rapportent les dires - Valls plaide pour la lutte contre les inégalités (35) -, tantôt elles-mêmes l'objet de critiques (34) :

(32) Notre défi, c'est la réduction des inégalités dans un environnement où il y a d'un côté les partisans du statu quo, qui refusent de faire évoluer notre modèle social, comme s'il était parfait, et de l'autre ceux qui pensent qu'il faut tout abattre et laisser le marcher réguler. (Le Figaro, 21-11-2015, interview avec L. Berger, CFDT) (33) «Cette loi a créé une situation illisible et injuste, a insisté Macron devant la commission spéciale. Nous voulons réduire les inégalités entre les salariés tout en rendant aux élus la liberté de s'adapter aux territoires. » (Libération, 26-01-2015)

(34) Quant à la réforme du collège, la dernière en date du gouvernement, elle [Lise, professeur de lettres] la juge «absurde dans ses objectifs affichés : réduire les inégalités [...] ». (Le Figaro, 25-07-2015)

(35) Pour venir à bout des maux qui rongent la société française, Valls plaide pour la lutte contre les inégalités (Le Figaro, 21-01-2015).

Enfin, on peut relever les actes de revendication de lutte contre les inégalités, présents dans les extraits ci-dessous, contenant des modalités déontiques.

(36) «François Hollande devrait revenir sur le terrain de la lutte contre les inégalités » assure un ministre. Il y a une partie de la France qui se sent oubliée, 
délaissée. C'est le moment de lui parler à nouveau, de faire des gestes. » (Le Figaro, 20-06-2015)

(37) Des politiques de l'offre, ajoutent nos auteurs, qui doivent être accompagnées d'une vigoureuse lutte contre les inégalités, tant une injuste répartition des revenus est un frein à la croissance. (Libération, 24-01-2015, chronique) part d'un journaliste ou d'un acteur de l'opposition, par exemple), annoncer ou faire des promesses de changement ( $\mathrm{du}$ côté des responsables politiques) et faire des revendications (de la part des journalistes et acteurs de l'opposition notamment) en faveur de l'amélioration en matière de lutte afin de parvenir à la réduction des inégalités. Si l'inégalité est souvent présentée comme un fait observable - un fait pluriel, concret et mesurable - ( $\$ 3.1)$, elle est aussi lieu d'action. Cette dimension ou ce «sème pragmatique » (Veniard 2013b : 33), [+ ce qui doit être combattu], s'inscrit également dans le fonctionnement sémantique du mot, qui semble "pousser" à l'action, aussi bien au sens d'acte de parole (critiquer, promettre, revendiquer) qu'au sens de mesures entreprises. Enfin, si la lutte dénote un procès, celui-ci est pourtant rarement en cours, mais plutôt prospectif: " "Les solutions, on ne les a pas aujourd'hui" ", dit, par exemple, quelqu'un dans l'entourage du premier ministre, lorsqu'il est question d'un «comité interministériel [qui] sera consacré en mars à des mesures de lutte contre les inégalités » (Le Figaro, 24-01-2015). Les mots projets, programme, réformes, moyens, mesures peuvent par ailleurs accompagner le thème de la lutte et situent ainsi le procès du côté de ce qui n'est pas encore accompli.

Par ailleurs, dans quelques-unes des constructions examinées, un certain degré de figement peut être observé, ce qui nous conduit à nous interroger sur la nature très convenue de cette revendication de lutte contre les inégalités, dont le caractère figé peut être le signe d'une certaine « langue de bois ».

\subsubsection{Une lutte à caractère très convenu...}

51 Le tableau ci-dessous montre des séquences de trois, voire quatre mots, comme le segment lutte contre les inégalités (55 occurrences dans le corpus) :

\begin{tabular}{|l|l|}
\hline Segments & Fréquences \\
\hline réduire les inégalités & 56 \\
\hline réduction des inégalités & 50 \\
\hline lutte contre les inégalités & 55 \\
\hline lutter contre les inégalités & 20 \\
\hline contre les inégalités & 103 \\
\hline
\end{tabular}

TABLEAU 4. Segments répétés et figement (Lexico3)

La récurrence ${ }^{23} \mathrm{du}$ segment lutte(r) contre les inégalités ainsi que la quasi-absence de structures modifiées du type «lutter efficacement contre les inégalités » confèrent à la séquence un caractère figé, voire de stéréotype discursif, préfabriqué, émergeant dans 
le discours médiatique qui tendrait vers un discours unificateur. Selon A. KriegPlanque, "[d]ans les représentations sociales, la "langue de bois" se rattache, à l'opposé d'une parole authentique, à diverses pratiques de préfabrication des discours mises au service de la propagande, des relations publiques, de la communication politique et publique » $(2017: 1)$. Dans les extraits suivants, l'hypothèse d'une langue de bois, "vide de sens ", à finalité "douteuse ${ }^{24}$, est même émise par les énonciateurs respectifs en (38) et (39):

(38) La lutte contre les inégalités est devenue une notion fourre-tout et une marque identitaire qui se coupe du réel et se moque des effets inverses qu'elle produit. (Le Figaro, 21-05-1015, débats)

(39) Le discours de Najat Vallaud-Belkacem, évoquant pêle-mêle les valeurs de l'école laïque, l'autorité, la lutte contre les inégalités, le décrochage scolaire et l'aide accrue aux enfants fragiles, ne pouvait que faire consensus. (Le Figaro, 23-01-2015)

(40) $\mathrm{Au}$ fil des pages reviennent les concepts et attitudes au cœur d'une "école refondée » - l'autonomie des élèves, la prise d'initiative, l'entraide, la curiosité, le plaisir et le sens de l'école. Un socle commun qui dépasserait le discours incantatoire de lutte contre les inégalités? (Le Monde, 14-02-2015)

(41) Face à un exécutif déterminé à imposer sa réforme du collège brandie comme un outil de lutte contre les inégalités, le syndicat classé à droite appelle à un blocage du brevet des collèges jeudi et vendredi. (Le Figaro, 24-06-2015)

Lutte contre les inégalités est employé en mention et caractérisé comme notion fourre-tout en (38). De plus, la séquence est évoquée en bloc, dans une série de concepts, parmi d'autres sujets (pêle-mêle), dans un discours qui fait consensus (39); elle enchante (40) caractéristique d'une langue de bois et d'un discours unificateur - et est caractérisée comme une stratégie, brandie par l'exécutif pour améliorer son image (41).

Le mot inégalité(s) tend ainsi vers ce que S. Bikialo et J. Rault (2018) appellent des « mots d'ordre $»^{25}$ : l'apport informationnel semble moindre et la revendication de la lutte contre l'inégalité, une « évidence », quel que soit l'énonciateur.

\section{Conclusion}

L'analyse du corpus de presse a permis de dégager trois dimensions sémantiques principales du mot inégalité(s) en discours, lequel fonctionne comme un fait concret et mesurable (dimension empirique), mais aussi comme un fait, qui évalué négativement (dimension injuste), peut être à la source de revendications (dimension pragmatique). Nous souhaitons revenir maintenant sur la façon dont ces trois tendances se manifestent dans la matérialité discursive et sur leurs interactions.

L'étude du cotexte d'inégalité(s) a permis de montrer que le mot renvoie, dans le corpus, à un fait pluriel, concret et mesurable. La focalisation se fait, avant tout, sur les inégalités multiples, et non pas sur l'inégalité en tant que notion abstraite; le mot fait référence à des situations qui se retrouvent dans la société et qui peuvent être observées, mesurées et chiffrées : inégalité(s) fonctionne alors, dans ces cas, comme un lieu d'observation. Cet usage spécifique du mot est à mettre en lien avec les pratiques des journalistes visant à aborder les questions sociales, comme celle de l'inégalité, à travers des éléments tangibles et quantifiables : en effet, on a relevé, dans l'entourage du mot, des données statistiques, provenant souvent de travaux de spécialistes dans le 
domaine, ainsi que des formes linguistiques exprimant l'intensité et la scalarité du phénomène.

Outre cette dimension empirique et "objectivisée », le mot peut aussi fonctionner comme un lieu d'évaluation : il comporte le sème [- positif], ce qui ouvre la voie vers des actes de critique et de revendication de la part de divers acteurs sociaux et politiques. Le segment lutte(r) contre les inégalités, qui apparaît notamment dans des articles de presse à tonalité subjective, participe à ces actes de parole; il fait alors inscrire un sème pragmatique dans le sens du mot: l'inégalité requiert effectivement une pratique, une action politique, celle de la lutte à son encontre.

(42) Grande cause d'«ambition nationale», le programme ["grand plan numérique » pour l'école] s'ouvre sur des accents guerriers : il s'agit de relever un défi, le « défi du numérique », et de livrer le plus juste des combats, la lutte contre les inégalités. (Le Monde, 01-01-2015)

(43) Pour le moment, les pistes ministérielles s'apparentent à de grandes déclarations de principe consensuelles. Les mesures s'articuleront autour de trois «axes prioritaires » : la laïcité, la réduction des inégalités et la mobilisation des partenaires de l'école. (Le Figaro, 18-01-2015)

Connotation négative - le mot étant associé à l'idée d'injustice - et dimension pragmatique - le mot appelant à une réponse active - se conditionnent mutuellement. Mais ce combat, la lutte contre pour la réduction des inégalités, se présente souvent, dans le discours de presse, comme une "évidence » partagée et consensuelle. Le vocabulaire guerrier, que l'on peut observer dans certains cotextes du mot et que relève également ce journaliste du Monde, ne qu'une autre illustration de l'effet rhétorique que peut revêtir une « langue de bois » en lien avec l'inégalité.

\section{BIBLIOGRAPHIE}

Europresse] Base de données de presse, http://www.europresse.com/fr/.

[Frantext] Base de données de textes français, ATILF-CNRS, https://www.frantext.fr/.

[Le Petit Robert] Rey-Debove J. \& Rey A. (dirs) (2012), Le Petit Robert. Dictionnaire alphabétique et analogique de la langue française, Paris, Le Robert.

[Le Trameur] Logiciel d'analyse automatique, statistique et documentaire de textes, S. Fleury, CLESTHIA, http://www.tal.univ-paris3.fr/trameur/.

[Lexico3] Logiciel de statistique textuelle, C. Lamalle, W. Martinez, S. Fleury \& A. Salem, SYLEDCLA2T, http://www.lexi-co.com/.

[TLF] Centre national de la recherche scientifique (dirs) (1983), Trésor de la langue française, Paris, Éditions du CNRS.

Austin J. L. ([1962] 1970), Quand dire, c'est faire, Paris, Seuil.

Bacot P., Desmarchelier D. \& Rémi-Giraud S. (2012), « Le langage des chiffres en politique », Mots.

Les langages du politique 100, p. 5-14. 
Bikialo S. \& Rault J. (2018), Au nom du réalisme. Usage(s) politique(s) d'un mot d'ordre, Paris, Éditions Utopia.

Bonnewitz P. (2015), Classes sociales et inégalités, Paris, Bréal.

Bosredon A. (1988), « Un adjectif de trop : l'adjectif de relation », Information grammaticale 37, p. 3-7.

Branca-Rosoff S. (2001), « La sémantique lexicale du mot "quartier" à l'épreuve du corpus Frantext ( $\mathrm{XII}^{\mathrm{e}}-\mathrm{XX}{ }^{\mathrm{e}}$ siècles) », Langage et société $96, \mathrm{p} .45-70$.

Cusin-Berche F. (1998), Le management par les mots. Étude sociolinguistique de la néologie, Paris, L'Harmattan.

Dubois J. (1962), Le vocabulaire politique et social en France de 1869 à 1872. À travers les œuvres des écrivains, les revues et les journaux, Paris, Larousse.

Duvoux N. (2017), Les inégalités sociales, Paris, PUF.

Fiala P. (1999), « Les termes de l'égalité et de l'inégalité : flux et reflux », dans Fiala P. (dir.), IN/ ÉGALITÉ/S. Usages lexicaux et variations discursives (18e $-20^{e}$ siècles), Paris, L'Harmattan, p. 7-20.

Flaux N. (1996), « Question de terminologie », dans Flaux N., Glatigny M. \& Samain D. (dirs), Les noms abstraits. Histoire et théories, Villeneuve-d'Ascq, Presses universitaires du Septentrion, p. 77-90.

Flaux N. \& Van de Velde D. (2000), Les noms en français : esquisse de classement, Paris, Ophrys. Gary-Prieur M.-N. (2011), Les déterminants du français, Paris, Ophrys.

Guérin O., Lecolle M. \& Veniard M. (2018), « Présentation », Langages 210, « Vers une sémantique discursive : propositions théoriques et méthodologiques », p. 5-16.

Guidée R. \& Savidan P. (dirs) (2017), Dire les inégalités : représentations, figures, savoirs, Rennes, Presses universitaires de Rennes.

Institut national de la statistique et des études économiques (2020), Tableaux de l'économie française, Insee Références, https://www.insee.fr/fr/statistiques/4318291, consulté le 20 août 2020.

Kerbrat-Orecchioni C. (2009), L'énonciation. De la subjectivité dans le langage, Paris, Armand Colin. Krieg-Planque A. (2017), « Langue de bois », Publictionnaire. Dictionnaire encyclopédique et critique des publics, http://publictionnaire.huma- num.fr/notice/langue-de-bois/, consulté le 16 avril 2019.

Lebart L. \& Salem A. (1994), Statistique textuelle, Paris, Dunod.

Martin R. (1996), « Le fantôme du nom abstrait », dans Flaux N., Glatigny M. \& Samain D. (dirs), Les noms abstraits. Histoire et théories, Villeneuve-d'Ascq, Presses universitaires du Septentrion, p. 41-50.

Mihatsch W. (2006), « Machin, truc, chose : la naissance de marqueurs pragmatiques », dans Drescher M. \& Frank-Job B. (dirs), Les marqueurs discursifs dans les langues romanes : approches théoriques et méthodologiques, Frankfurt am Main, Peter Lang, p. 153-172.

Moirand S. (2011), « Du sens tel qu'il s'inscrit dans l'acte de nommer », dans Dahlet V. B. (dir.), Ciências da linguagem e didática das línguas, São Paulo, Humanitas, FAPESP, p. 165-179.

Moirand S. (2016), « De l'inégalité objectivisée à l'inégalité ressentie et aux peurs qu'elle suscite : les réfugiés pris au piège de l'identité », Revista de Estudos da Linguagem 24 (3), p. 1015-1046, 
http://www.periodicos.letras.ufmg.br/index.php/relin/article/view/10812, consulté le 16 avril 2019.

Née É. (2012), L’Insécurité en campagne électorale, Paris, Honoré Champion.

Paveau M.-A. (2017), « Le discours des vulnérables. Proposition théorique et politique », Cadernos de Linguagem e Sociedade 18 (1), p. 135-157,

http://periodicos.unb.br/index.php/les/article/view/1571, consulté le 16 avril 2019.

Péroz P. (2002), « La préposition contre à partir de la valeur "d'échange". Variation sémantique et régularité des opérations linguistiques », Scolia 15, p. 107-122.

Savidan P. (2006), «Inégalités, équité et justice sociale », dans Mesure S. et Savidan P. (dirs), Le dictionnaire des sciences humaines, Paris, Quadrige / PUF, p. 626-629.

Sebbah B., Souillard N., Thiong-Kay L. \& Smyrnaios N. (2018), Les Gilets Jaunes, des cadrages médiatiques aux paroles citoyennes, rapport de recherche préliminaire,

http://universitepopulairetoulouse.fr/spip.php?article1524, consulté le 27 février 2019.

Sériot P. (1986), «Langue russe et discours politique soviétique : analyse des nominalisations », Langages 81, p. 11-41.

Tournier M. (1996), « Français à l'extrême-droite, un mot habité », dans Rémi-Giraud S. \& Rétat P. (dirs), Les mots de la nation, Lyon, Presses Universitaires de Lyon, p. 65-81.

Veniard M. (2013a), La nomination des événements dans la presse. Essai de sémantique discursive, Besançon, Presses universitaires de Franche-Comté.

Veniard M. (2013b), « Du profil lexico-discursif du mot "crise" à la construction du sens social d'un événement », dans Londei D., Moirand S., Reboul-Touré S. \& Reggiani L. (dirs), Dire l'événement. Langage, mémoire, société, Presses Sorbonne Nouvelle, p. 221-232.

\section{NOTES}

1. Le cas récent du mouvement des Gilets jaunes, qui, en novembre 2018, a fait irruption dans la scène publique française, en est d'ailleurs une illustration; la question des inégalités sociales et fiscales apparaît, en effet, comme l'une des raisons principales du mécontentement des manifestants (cf. Sebbah et al. 2018). La question apparaît aussi dans le traitement médiatique de la crise sanitaire provoquée par la pandémie de Covid-19, comme l'illustrent ces titres d'article : «Covid-19, miroir des inégalités territoriales et sociales dans le 93 » (Libération.fr, 05-03-2020); "Jacques Toubon: "Les inégalités deviennent encore plus criantes avec l'état d'urgence sanitaire" " (LeMonde.fr, 01-04-2020).

2. Depuis la publication en 1755 par J.-J. Rousseau de son Discours sur l'origine et les fondements de l'inégalité parmi les hommes, le débat autour de l'inégalité s'est progressivement enrichi des échanges multiples entrepris notamment en philosophie, en science économique et en sociologie. À l'époque contemporaine, la question de l'inégalité apparaît souvent en résonance avec d'autres notions, comme celles d'équité et de justice sociale ; les analyses sociologique et économique, qui, dans les années 1960 et 1970, ont centré l'explication des inégalités sur la notion de classe sociale, s'attachent aujourd'hui à étudier également les inégalités ethno-raciales, de genre, de religion ou d'orientation sexuelle (cf., pour un aperçu de ces approches, Bonnewitz 2015 ; Duvoux 2017 ; Savidan 2006). D'autres études proposent de saisir et de questionner la thématique à travers les manières (perceptions, émotions, sentiments, idéaux, représentations) de dire l'inégalité (Guidée \& Savidan 2017); elles peuvent aussi s'inscrire dans une perspective théorique prenant en 
compte la question du pouvoir, sous les formes multiples de violence et de domination (Paveau 2017).

3. Ce travail s'inscrit dans le prolongement d'une étude initialement présentée lors du IV Symposium international sur l'analyse du discours (SIAD), qui a eu lieu les 14-17 septembre 2016, à Belo Horizonte, au Brésil, et qui portait sur l'articulation entre inégalités sociales et discours.

4. Logiciels d'analyse lexicométrique et textométrique développés à l'université Sorbonne nouvelle - Paris 3 dans le cadre du CLESTHIA (EA 7345).

5. Mihatsch (2006).

6. Un segment répété est une suite de formes dont la fréquence est égale ou supérieure à deux dans le corpus (Lebart \& Salem 1994 : 317). Cette démarche permet d'observer une forme précise dans son entourage immédiat.

7. Le fonctionnement d'inégalité(s) rejoint, sur plusieurs aspects, celui de crise (Veniard 2013b) et celui d'insécurité (Née 2012). Sur le plan référentiel en particulier, ces trois mots se caractérisent par la multiplication des domaines dans lesquels ils peuvent apparaître : crise économique, sociale, financière; insécurité routière, sanitaire, etc.

8. Les exemples cités ont été extraits des dictionnaires consultés, entrée « inégalité ».

9. Journal officiel de la Commune, exemplaire du 31 mars 1871.

10. Cette idée, qui était déjà présente dans les études discursives des années 1960-1970, a été remise en lumière par des chercheur.es en analyse du discours et en lexicologie au tournant des années 2000 (Branca-Rosoff 2001, Cusin-Berche 1998 ; cf., à ce propos, Veniard 2013a).

11. Selon N. Flaux, « alors que le passage au pluriel n'entraîne généralement pas de changement de sens pour les noms concrets, (un chien / des chiens), il en va différemment pour les noms abstraits, du moins pour ceux qui admettent le pluriel. Dans de nombreux cas, l'emploi au pluriel s'accompagne d'un changement de sens » (1996:89).

12. Nous avons constaté que, dans au moins $62,7 \%$ des occurrences d'inégalité(s), la détermination du nom se fait par des définis (l'inégalité: 147 occurrences; les inégalités : 948 occurrences). La forme est donc majoritairement utilisée au pluriel et s'accompagne préférentiellement d'un article défini.

13. Le corpus utilisé pour cette exploitation est constitué des textes disponibles sur la base Frantext parus depuis le XIII ${ }^{\mathrm{e}}$ siècle. Pour le relevé quantitatif des occurrences, nous avons pris en compte l'ensemble des formes graphiques d'inégalité(s). Consultation réalisée en juin 2019.

14. L'extrait qui suit illustre la présence, dans le corpus, de cet aspect du mot : « Il [T. Piketty] a été le premier à observer les distributions statistiques par centiles $(1 \%)$, voire par tranches de $0,1 \%$ (un millième de la population). Au contraire, l'Insee continue de raisonner par déciles, notamment pour mesurer les inégalités, véritable obsession française (le rapport de 3,5 entre le décile des plus riches et celui des plus pauvres est stable)»(Le Figaro, 05-10-2015; nous soulignons en italiques).

15. Dans le corpus, la réduction des inégalités (l'idée de diminution) apparaît, le plus souvent, dans des structures exprimant un souhait, ou une revendication. La baisse de l'inégalité se situant plutôt dans le domaine du prospectif, et non pas dans celui de l'observable, nous l'avons traitée dans la section consacrée à l'analyse du mot en tant que support de revendications ( $\$ 3.3$, infra).

16. P. Sériot, dans son étude des discours politiques soviétiques, a montré comment la nominalisation peut renvoyer à du préconstruit, à du "déjà-là »: "l'énoncé nominalisé est préconstruit, c'est-à-dire qu'il n'est pas pris en charge par le sujet énonciateur, mais se trouve comme un objet du monde "déjà là", préexistant au discours » (1986:24). L'effet produit par la nominalisation est celui d'une évidence ; l'information est présentée comme allant de soi.

17. En effet, on l'a vu, la représentation des inégalités a évolué au cours de l'histoire et des événements : « Octroyer des privilèges liés à la naissance apparaît aujourd'hui comme scandaleux alors que la société d'ordres était fondée sur ce critère », rappelle P. Bonnewitz (2015 : 21). 
18. La notion de cooccurrence rend compte de «la présence simultanée, mais non forcément contiguë, dans un fragment de texte (séquence, phrase, paragraphe, voisinage d'une occurrence, partie du corpus etc.) des occurrences de deux formes données » (Lebart \& Salem 1994 : 312). Le calcul des cooccurrents d'une forme pôle peut s'appuyer sur un indice de spécificité : une forme $i$ est dite "spécifique positive » de la partie $j$ quand sa fréquence réelle, dans cette partie, est supérieure à la fréquence attendue (ibid. : 318).

19. Nous reprenons ici l'idée de P. Péroz selon laquelle la préposition contre marque la réduction de l'« altérité constitutive », c'est-à-dire la réduction de la différence de localisation, des termes qu'elle met en relation (2002: 118). Dans le cas où contre prend la valeur d'hostilité, cette réduction passe par la disparition de l'un des termes de la relation (ibid. : 121).

20. Comme en témoigne le tollé d'indignation qu'a suscité la revendication explicite d'A. Petit, président du CNRS, en faveur d'une loi «darwinienne » et « inégalitaire » dans le cadre de la future loi de programmation pluriannuelle de la recherche (Libération, 12-12-2019, "Loi darwinienne" : au CNRS, le torchon brûle entre le PDG et les scientifiques »).

21. Actrice ou acteur politique ou membre de la société civile.

22. Au sens de J. L. Austin (1970).

23. Dans son travail sur le mot insécurité dans Le Monde, durant la campagne électorale de 2001-2002, É. Née (2012) a également constaté une fréquence élevée du segment lutte contre l'insécurité.

24. De fait, « dans le sentiment rhétorique commun, les caractéristiques les mieux partagées de la "langue de bois" portent sur sa finalité et sur ses effets. Du côté de sa finalité, la "langue de bois" est perçue comme visant à tromper, manipuler, dissimuler, travestir, faire diversion... [...]» (Krieg-Planque 2017 : 1). Selon la définition du Petit Robert, la langue de bois désigne la « façon de s'exprimer qui abonde en formules figées et en stéréotypes non compromettants » (entrée « bois »; nous soulignons en italiques).

25. Les auteurs envisagent le mot réalisme comme un mot d'ordre : utilisé par les femmes et les hommes politiques de façon courante, le mot s'accompagne de l'injonction, présentée comme évidente, de se conformer à ce que dicte la réalité (Bikialo \& Rault 2018).

\section{RÉSUMÉS}

L'inégalité est une thématique sociale, abordée de façon récurrente dans les médias. Appliquant une approche de "sémantique discursive", nous examinerons l'usage d'inégalité(s) dans le discours de presse en circonscrivant les principales dimensions sémantiques du nom à travers ses fonctionnements préférentiels en cotexte, étroit ou élargi. Après quelques questionnements sémantico-référentiels autour du mot, l'étude du corpus - constitué d'articles issus des journaux Le Monde, Libération et Le Figaro - montrera qu'inégalité(s) fonctionne, à la fois, comme un lieu d'observation, d'évaluation et de revendication. L'analyse conduit également à penser que l'usage partagé et consensuel du segment lutte contre les inégalités dans le corpus fait apparaître une forme de « langue de bois».

Inequality is a common social theme which arises regularly in the press. Using a semanticdiscursive approach, we will examine the use of the word inégalité(s) (inequality(s)) in media discourse with the aim to circumscribe the main semantic dimensions of the word through its functioning in co-text. After some semantical and referential considerations, data analysis will 
show that inégalité(s) functions as lieu of observation, evaluation and political claims. The corpus contains newspaper articles from Le Monde, Libération and Le Figaro. The data analysis also lead to the assumption that the shared and consensual use of the segment lutte contre les inégalités (fight against inequality) shows features of some sort of doublespeak ("langue de bois").

INDEX

Mots-clés : analyse du discours, sémantique, discours de presse, inégalité(s), énonciation

Keywords : discourse analysis, semantics, media discourse, inequality, enonciation

\section{AUTEURS}

PASCALE BRUNNER

Université de Poitiers - FoReLLIS

\section{MICHELE PORDEUS-RIBEIRO}

CNPq - Universidade de São Paulo et CLESTHIA-Cediscor \& Diálogo 